\title{
Counting All the Antistars in the Sky
}

\section{Analyzing gamma-ray sources leads to an upper limit on how many antimatter stars could exist in the Milky Way.}

\section{By Rachel Berkowitz}

$\mathrm{N}$ owadays it is taken for granted that the Universe contains no substantial amounts of antimatter. Most cosmological models include hypothetical physical processes to explain why matter dominates although the Universe should have been created with equal amounts of both. But in 2018, the Alpha Magnetic Spectrometer 2 (AMS-02) experiment on the International Space Station possibly detected several antihelium nuclei, suggesting that some original antimatter survived to form antistars and even antigalaxies. Now, Simon Dupourqué and colleagues at the Institut de Recherche en Astrophysique et Planétologie (IRAP), France, identify possible antistars based on ten years of gamma-ray observations from the orbiting Fermi Gamma-ray Space Telescope and derive constraints on how many such objects might exist in our solar neighborhood [1].

Gamma rays are produced when a particle and its antiparticle collide and annihilate. On an antistar, this process is thought to occur when regular interstellar matter accretes onto the antistar's surface. But many other astrophysical phenomena

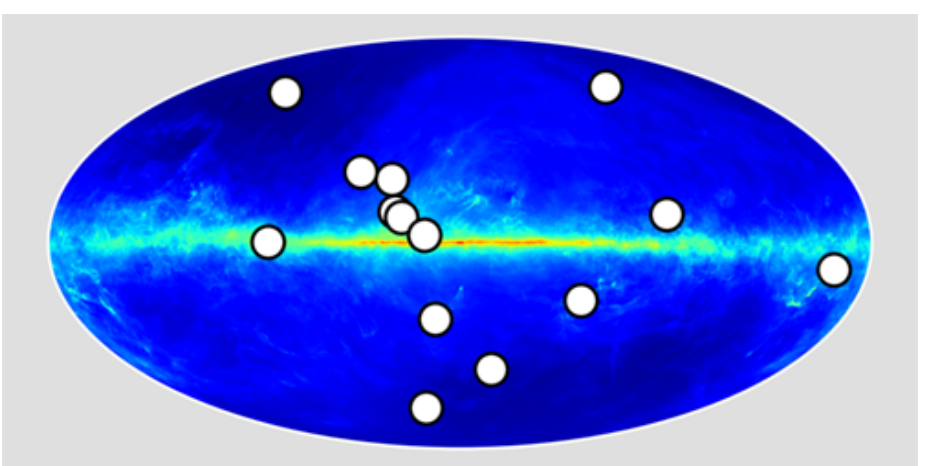

Credit: S. Dupourqué/IRAP can also emit gamma rays, with each source exhibiting a characteristic spectrum and light curve. To identify possible antistars among the 5787 gamma-ray sources cataloged by the Fermi mission, Dupourqué and his colleagues calculated which sources have a point-like geometry and a spectrum compatible with baryon-antibaryon annihilation. Combining their calculations with simulations of the accretion process around antistars, the researchers derived an upper limit of 2.5 antistars per million normal stars within several hundred light years from our Sun-assuming the antistars have properties similar to normal stars.

While a single nearby antistar might have produced the possible AMS-02 antihelium, the researchers suggest a more likely source outside of the main Galactic Disk, where antistars could more easily "hide" from gamma-ray detection.

Rachel Berkowitz is a Corresponding Editor for Physics based in Vancouver, Canada.

\section{REFERENCES}

1. S. Dupourqué et al., "Constraints on the antistar fraction in the Solar System neighborhood from the 10-year Fermi Large Area Telescope gamma-ray source catalog," Phys. Rev. D 103, 083016 (2021). 